\title{
Langmuir
}

This document is confidential and is proprietary to the American Chemical Society and its authors. Do not copy or disclose without written permission. If you have received this item in error, notify the sender and delete all copies.

\section{Mechanistic insights into the directed assembly of hydrogel blocks mediated by polyelectrolytes or microgels}

\begin{tabular}{|r|l|}
\hline Journal: & Langmuir \\
\hline Manuscript ID & la-2017-00924f \\
\hline Manuscript Type: & Article \\
\hline Date Submitted by the Author: & $17-$ Mar-2017 \\
\hline Complete List of Authors: & $\begin{array}{l}\text { Hanauer, Nicolas; Universite de Montreal } \\
\text { Latreille, Pierre Luc; Universite de Montreal } \\
\text { Banquy, Xavier; Universite de Montreal, Faculty of Pharmacy }\end{array}$ \\
\hline
\end{tabular}

SCHOLARONE ${ }^{m}$

Manuscripts 
1 Mechanistic insights into the directed assembly of hydrogel blocks mediated

2

3

4

5

6

7

8

\title{
by polyelectrolytes or microgels
}

\author{
Nicolas Hanauer, Pierre Luc Latreille, Xavier Banquy* \\ Canada Research Chair in Bio-inspired Materials and Interfaces, Faculty of Pharmacy, Université de Montréal \\ C.P. 6128, succursale Centre Ville, Montréal, QC H3C 3J7, Canada \\ *corresponding author: xavier.banquy@umontreal.ca
}

\begin{abstract}
:
In this study, we report the directed assembly of hydrogel blocks mediated by electrostatic interactions. We compared two different assembly mechanisms, one mediated by microgel particles and another mediated by direct interaction between oppositely charged blocks. The system consisted in hydrogel blocks made of an interpenetrated network of (hydroxyethyl)methacrylate-poly(ethyleneglycol)dimethacrylate (HEMA-PEGDMA) and either positively charged polyethyleneimine (PEI) or negatively charged hyaluronic acid (HA). Positively charged hydrogel blocks were pretreated with negatively charged microgel particles (MG) made of $\mathrm{N}$-isopropylacrylamide-methacrylic acid. Both systems (PEI/HA and $\mathrm{PEI} / \mathrm{MG}$ ) demonstrated spontaneous directed assembly, meaning that positive blocks were systematically found in contact with oppositely charged blocks. Directed assembly in water of $\mathrm{PEI} / \mathrm{HA}$ blocks resulted in large and open aggregates while PEI/MG blocks exhibited more compact aggregates. Effects of salt and $\mathrm{pH}$ were also assessed for both systems. Inhibition of blocks aggregation was found to appear above a critical salt concentration $\left(C_{\text {Salt }}^{*}\right)$ which was significantly higher for the PEI/HA system $(80 \mathrm{mM})$ compared to the PEI/MG system (5$20 \mathrm{mM}$ ). The observed difference was interpreted in terms of the nanostructure of the contact area between blocks. Blocks aggregation was also found to be controlled by the content of
\end{abstract}


25 negatively charged groups in the microgels as well as the concentration of MG in the

26 suspension $\left(C_{M G}\right)$ used to treat the hydrogel block surfaces. Our results shine light on the

27 subtle differences underlying the adhesion mechanisms between hydrogel blocks and suggest

28 new routes toward the design of innovative complex soft materials.

29 Keywords: hydrogel block, microgels, polyelectrolytes, directed assembly, adhesion 30 
31

32

33

34

\section{Introduction}

Hydrogels, composed of a polymeric matrix and an "immobilized" liquid phase, are ideal materials for bioengineering ${ }^{1}$. On one hand, their polymeric structure is highly versatile and tunable in terms of physical (swelling, stiffness, porosity...) and chemical modifications (functionalizations, sensitivity to environmental cues...). On the other hand, the trapped liquid phase can be used to load and preserve different active compounds (chemical species, growth factors, cells....) in the polymeric network. Since hydrogel matrices are highly tunable, they offer the possibility to design matrices with finely tuned structural environment which in turn can direct the fate of the species they carry ${ }^{2}$. These unique properties have initially been used to develop cargos for drug delivery systems ${ }^{3,4}$. For example, cell-seeded hydrogel scaffolds with various internal cues are now the prime techniques used for the regeneration of a large

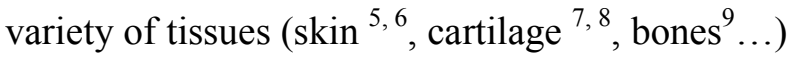

Researchers have extensively used assembly techniques to gain a finer control over the microenvironment inside these hydrogel matrices ${ }^{10}$. In situ gelation is the easiest path to follow since many different polymeric interactions can lead to the hydrogel formation such as chemical crosslinking ${ }^{11,12}$, electrostatic interactions ${ }^{13,14}$ or complementary binding ${ }^{15,16}$. In the biomedical field, hydrogel formation is triggered by an external stimulus (temperature, $\mathrm{pH} . .$. ) upon injection of the reactive components. This approach can be limited by physiological conditions, toxicity of the injected components or by the poor control over the hydrogel structure.

An emerging approach to obtain in situ hydrogel matrices with controlled architecture is using the directed assembly of prefabricated hydrogel blocks ${ }^{17,18}$. This bottom-up approach could possibly offer a better control over the three dimensional distribution of embedded active compounds. It might also unlock new possibilities of combining a large spectrum of mechanical and biochemical cues within a defined hydrogel scaffold. This would simply 
56 require selecting formulations and functionalizations needed in separate blocks to design the

57 desired scaffold. It might also be a powerful approach to program a predefined structure to 58 mimic the bio-functional organization of a tissue.

59 Different experimental techniques have been proposed to facilitate the directed assembly of 60 hydrogel blocks. Blocks aggregation via entropic constraints (such as confinement or 61 concentration increase) can lead to the formation of aggregates but the lack of control over 62 blocks organization and mechanical integrity is still a concern ${ }^{19}$. Microfluidic devices have 63 demonstrated great potential to produce selective aggregation of a few blocks but their ability 64 to produce large scale tissues-sized, aggregates seems more difficult to adapt ${ }^{20,21,22}$. 65 Stabilization of blocks in water-in-oil droplets followed by secondary photo crosslinking has 66 allowed a fine control over the size of the blocks aggregates but not over their final internal 67 structure ${ }^{23}$. Using templates with hydrophobic and hydrophilic regions is also a method to 68 guide blocks organization on a patterned surface ${ }^{24}$ but appears to be challenging to translate 69 into 3D structures. Tissue printing is certainly the most promising solution towards 3D 70 organization of cell-laden hydrogels is extensively used to obtain layered materials rather than 71 injectable blocks ${ }^{25}$. Other fabrication techniques have also been proposed via the 72 incorporation of magnetic cues to guide the blocks assembly ${ }^{26}$.

73 Nevertheless, since hydrogels are easily tunable, the most promising approach to obtain 74 scaffolds with programmable internal structures is based on block-block interactions, 75 especially to promote directed assembly. Hydrogel blocks can be designed to interact and 76 adhere specifically with neighboring blocks. A wide spectrum of adhesive mechanisms have 77 been tested for that purpose: Michael type addition between reactive groups at blocks 78 surfaces $^{27}$, molecular recognition via host-guest interactions ${ }^{28,29}$, complementary DNA chains 79 incorporated in the hydrogel blocks ${ }^{30}$, nucleation and growth of collagen fibers at interfaces

$80{ }^{31}$. These techniques make use of a wide variety of adhesion mechanisms, resulting in 
81 complex, structurally controlled hydrogel assemblies. Furthermore, it has been demonstrated

that surface modification with polymers brushes or nanoparticles can also efficiently promote adhesion between soft surfaces ${ }^{32,33,34,35}$. Besides the large body of work demonstrating the potential uses of structurally programmed matrices, there is a lack of systematic studies comparing their assembly mechanism and sensitivity to external physical factors. Such knowledge is critical to promote the development of more complex materials integrating a large number of chemical, structural and physical characteristics.

In this report, we have intended to rationalize the assembly of hydrogel blocks mediated by electrostatic interactions. We studied two different interaction mechanisms, one where hydrogel blocks assembly is mediated by direct contact between oppositely charged blocks and a second mechanism where assembly between identical blocks is mediated by oppositely charged microgel particles (MG). The hydrogel blocks were fabricated by UV photolithography in presence of different polyelectrolytes (PEI or HA). Since electrostatic forces were expected to drive the blocks assembly, we studied the effect of $\mathrm{pH}$, ionic strength and microgel composition to elucidate differences between the two interaction mechanisms.

\section{Materials and methods:}

Materials

2-hydroxyethyl methacrylate (HEMA, 97\%), poly(ethylene glycol)dimethacrylate $($ PEGDMA, $\quad \mathrm{Mn}=550 \mathrm{~g} / \mathrm{mol}), \quad \mathrm{N}, \mathrm{N}^{\prime}$-methylenebis(acrylamide) $\quad($ BisA, $99 \%), \quad \mathrm{N}-$ Isopropylacrylamide (NIPAM, >99\%), sodium dodecyl sulfate (SDS, $>98.5 \%$ ), methacrylic acid (MAA, 99\%) hydrochloric acid $(\mathrm{HCl}, 37 \%)$ and aluminum oxide (activate, basic, Brockmann I) were purchased from Sigma-Aldrich Canada, Ltd. (Oakville, Canada). Irgacure 2959 was a kind gift from BASF (Mississauga, Canada). Polyethyleneimine (PEI, branched, $\mathrm{Mw}=10000 \mathrm{~g} / \mathrm{mol}, 99 \%$ purity) was from Alfa Aesar (Ward Hill, USA). Sodium hyaluronate 
106 (HA, Mw $=60000 \mathrm{~g} / \mathrm{mol}$ ) was purchased from LifeCore Biomedical (Chaska, USA). Sodium

107 chloride $(\mathrm{NaCl})$ and ammonium persulfate (APS) were from Fisher Chemical (Ottawa,

108 Canada). Unless stated, materials were used without prior purification.

109 Hydrogel blocks preparation

110 Blank hydrogel blocks were obtained via photopolymerization of HEMA using PEGDMA as

111 a cross-linker. After purification on a basic aluminum oxide column, a mixture of HEMA-

112 PEGDMA (99.8:0.2 mol\%) was dissolved in water (65 wt \%) for $10 \mathrm{~min}$ under magnetic

113 stirring. Irgacure 2959 was then added as photoinitiator (5 $\mathrm{wt} \%$ total). The mixture was then

114 placed under high intensity UV lamp (UVP Mercury Spot Low, 100MW Longwave) for 30

$115 \min$.

116 Hydrogel solutions were injected in a mold composed of two glass slides separated by a glass

117 spacer. The loaded mold was then covered with a photomask and another glass slide. The

118 photomasks consisted of a transparent slide imprinted with the periodic arrangement of the

119 blocks shape. With this technique, we were able to obtain hydrogel blocks of size larger than

$1201 \mathrm{~mm}$ and thickness ranging between $0.15 \mathrm{~mm}$ to $3 \mathrm{~mm}$ depending on the thickness of the

121 spacer used. After polymerization, the injection mold was opened, the unreacted mixture was

122 washed away with water under pressure and the blocks were gently separated from the glass

123 plates with a spatula. Blocks were then kept in water $(\sim 25$ blocks / 10mL $)$ under high

124 magnetic stirring to ensure complete swelling and removal of unreacted monomers.

125 Positively charged hydrogel blocks were obtained by adding polycationic PEI (25 wt $\%$ ) prior

126 to the photopolymerization step. PEI was added to the HEMA-PEGDMA mixture and then

127 dissolved in water at around $40^{\circ} \mathrm{C}$, allowing for the complete dissolution of PEI. PEI blocks

128 were colored in red by adding a few droplets of a Rhodamine $6 \mathrm{G}$ solution $(0.5 \mathrm{mg} / \mathrm{mL})$ in the

129 monomer mixture before polymerization. Similarly, in order to obtain negatively charged

130 blocks, an anionic polyelectrolyte was added to the reagents mixture prior to 
131 photopolymerization. A HA solution $(3 \mathrm{mg} / \mathrm{mL})$ was prepared one day prior to 132 photopolymerization in a water and $\mathrm{HCl}$ mixture (6.6 vol\%). This $\mathrm{HA}$ solution was used to 133 mix the monomer solution of HEMA and PEG-DMA at a final concentration of $35 \mathrm{wt} \%$. HA 134 blocks were colored in blue using a food coloring dye before polymerization.

135 The second procedure used to produce negatively charged blocks was to treat positively 136 charged blocks with a solution of negatively charged NIPAM-MAA microgels. Blocks were 137 immersed in a $\mathrm{MG}$ solution at $C_{M G}=4 \mathrm{mg} / \mathrm{mL}$ (25 blocks/10mL, 24 hours) under magnetic 138 stirring and then rinsed in water for 1 hour to eliminate non adsorbed microgels.

139 Microgels synthesis

140 NIPAM-MAA microgels used in this study were synthesized by precipitation polymerization. 141 Briefly, monomers (NIPAM with MAA at $0,5,10$ or $20 \mathrm{~mol} \%$ ), BisA as cross-linker (5mol\% 142 total monomers) and SDS $(867 \mu \mathrm{mol} / \mathrm{L})$ as surfactant were dissolved in degassed water. The 143 mixture was then placed at $65^{\circ} \mathrm{C}$ under mechanical stirring $(200 \mathrm{rpm})$ and argon atmosphere 144 for equilibration. APS (2.9 mmol/L) was then injected in the reaction vessel. Polymerization 145 was let to proceed during $4 \mathrm{~h} 30$ at a temperature of $75^{\circ} \mathrm{C}$ and constant stirring of $300 \mathrm{rpm}$. The 146 resulting particle solution was then dialyzed (Spectra/Por Tube-A-Lyzer Dynamic Dialysis 147 Device, $100 \mathrm{kDa}$ MWCO) against milliQ water $(\sim 60 \mathrm{~mL}$ of particle suspension for $20 \mathrm{~L}$ of 148 water, overnight). Four batches of microgels were synthesized containing 0 to $20 \%$ of MAA. 149 The concentration of microgels, $C_{M G}$, in the final suspension was determined by lyophilizing a 150 volume of $1.5 \mathrm{~mL}$ of suspension. Size, polydispersity and $\zeta$ potential of the MG particles $151(100-800 \mu \mathrm{g} / \mathrm{mL})$ in water and in presence of different salt concentrations at $22^{\circ} \mathrm{C}$ were 152 characterized via dynamic and phase analysis light scatterings (DLS and PALS) using a 153 Brookhaven NanoBrook Omni (90 detection angle, illumination wavelength $640 \mathrm{~nm})$. The 154 microgels zeta potential was found to be negative independently of the MAA content. Since 155 the polymerization of NIPAM was initiated by ammonium persulfate which is negatively 
156 charged in solution, the polymer chain ends bearing the initiator moiety are expected to

157 provide negative charges at the surface of the microgel even at $0 \%$ of MAA.

158 Directed assembly tests

159 Tests were performed in small crystallizers filled with 10 mLof distilled water. Mixtures of 160 positively and negatively charged $2 \times 2 \times 1 \mathrm{~mm}^{3}$ blocks were suspended together under constant

161 mixing conditions with an orbital shaker (150-200 RPM, 3min) until completion of the 162 assembly process. Upon completion of the assembly process, aggregates were imaged and 163 counted under a stereoscopic microscope (Zeiss Stereo Discovery V8 stereomicroscope). 164 Each assembly test was carried out in quintuplet and data were reported as mean value \pm 165 standard deviation. Cycles of assembly and disassembly were performed during each test to 166 evaluate surface integrity and directed assembly reproducibility. Once an aggregation test was 167 performed, the cubes were gently separated using a spatula before repeating next assembly 168 test. When the adhesion between the cubes was weak, gentle manipulation without inserting 169 the spatula in between the cubes was sufficient to separate them.

170 The effects of $C_{\text {Salt }}$ and $\mathrm{pH}$ on the aggregation process were also studied. Salinity of the 171 media was controlled using $\mathrm{NaCl}$. Blocks were left 10 min or 24 h to equilibrate in a $\mathrm{NaCl}$ 172 solution $(25$ blocks $/ 10 \mathrm{~mL})$ before testing their assembly in freshly prepared saline medium.

173 Assembly tests under acidic or basic conditions were achieved using a similar protocol with $174 \mathrm{HCl}$ and $\mathrm{NaOH}$ solutions $(\mathrm{pH}=3$ and $10.5,24 \mathrm{~h}$ equilibrium). Imaging of the blocks and 175 aggregates was performed on a Zeiss Stereo Discovery V8 stereomicroscope under high 176 illumination. During observation, the samples were immersed in water in a small crystallizer. 177 


\section{Results}

180

181

182

183

184

185

186

187

188

189

190

191

192

Aggregation number:

\section{HA PEI}

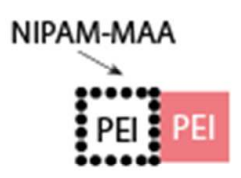

4
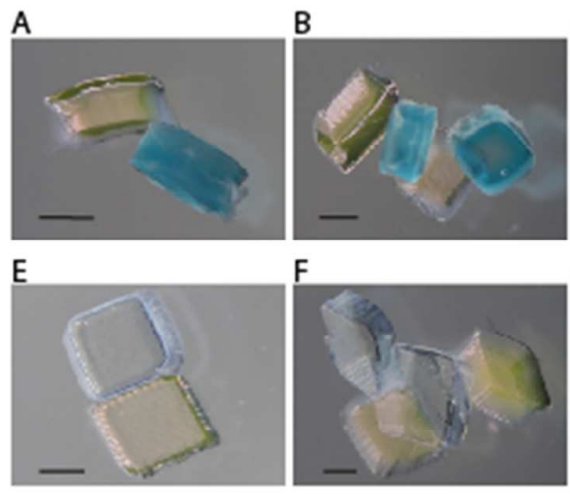

A
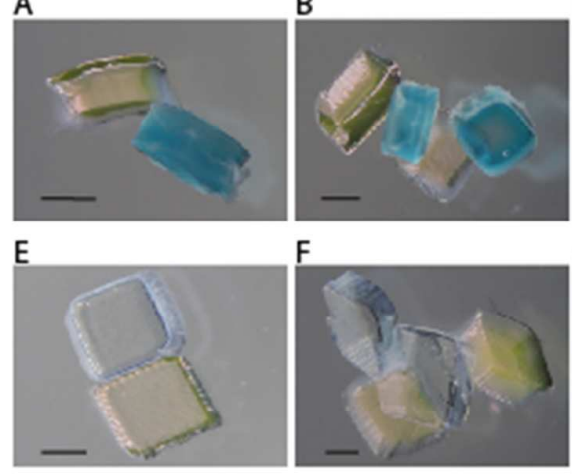

6
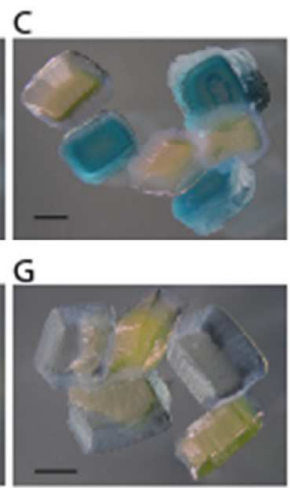

10

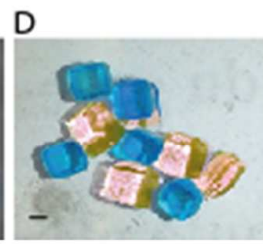

$\mathrm{H}$

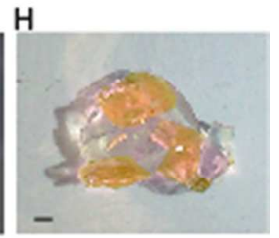

Figure 1: Microscopy images of PEI/HA (A-D) and PEI/MG (E-H) aggregates of different sizes (scale bar: $1 \mathrm{~mm}$ ) obtained after completion of the aggregation process. 
194 Both studied systems were tested under different conditions to investigate their properties and

195 differences. In a first series of test, we studied the effect of the population size for the PEI/HA

196 system, i.e. the effect of blocks concentration on aggregates size (maintaining the ratio

197 between block partners equal to 1). We studied three PEI/HA blocks populations: 3:3, 5:5 and

$19810: 10$ by performing 5 iterations of the aggregation test with the same blocks. For the three

199 tested populations, the average aggregates size was significantly reduced after the first

200 aggregation test and continued to gradually decrease until 5 iterations were performed (See

201 Figure 2).

202 We observed that an increase of the block population size led to larger aggregates at the first

203 iteration (the cumulative percentage reaches $100 \%$ at a high aggregation number). For

204 example, the 10:10 population exhibited large aggregates only ( $>17$ blocks) after the first

205 aggregation test while the 3:3 and 5:5 populations lead to a mixture of mid-sized aggregates

206 (4 to 6 blocks per aggregates).
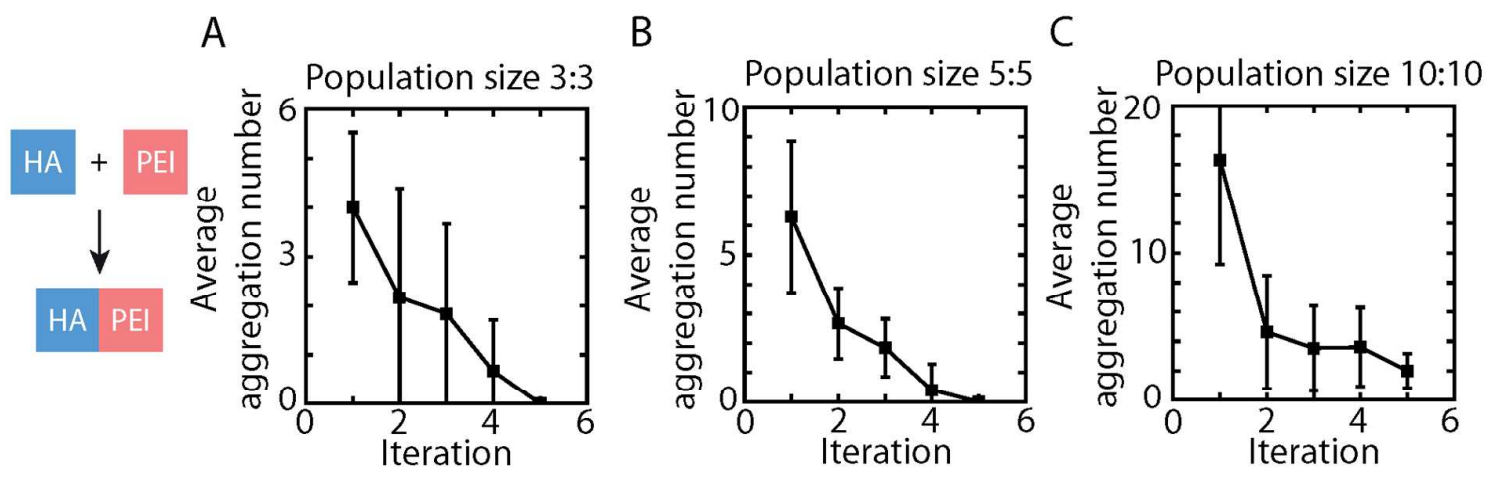

Figure 2: Average aggregation number as a function of the experiment iteration for different blocks concentrations. Error bars represent the standard deviation of 5 separate experiments.

In the next series of experiments, we compared the aggregation processes of our two systems.

209 For the PEI/MG system, PEI blocks pretreated with MG were mixed with equal number of

210 untreated PEI blocks. We tested 4 types of MG containing increasing amount of MAA (See

211 Table 1) and studied the directed assembly of the hydrogel blocks after pretreatment with

212 these microgels $\left(C_{M G}=4 \mathrm{mg} / \mathrm{mL}, 25\right.$ blocks / 10mL, 24 hours $)$. We used a block population 
213 size of 5:5 and performed 3 consecutive assembly/disassembly iterations for each test (see 214 Figure 3).

215 We observe that, while pretreatment with microgels containing MAA $0 \%$ did not lead to any 216 aggregation, pretreatments with the three other types of microgels (MAA 5\%, 10\% and 20\%) 217 lead to the formation of large aggregates at the first iteration (with 8 to 10 blocks per 218 aggregate). Increasing the number of assembly/disassembly cycles slightly decreased the 219 aggregates size but did not inhibit their formation in contrast to our previous observations 220 with the PEI/HA blocks.

Table 1: Particle size and $\zeta$-potential of the NIPAM-MAA microgels

\begin{tabular}{ccc}
\hline MAA $\%$ & $\mathrm{~d}(\mathrm{~nm})$ & $\zeta$-potential $(\mathrm{mV})$ \\
\hline $0 \%$ & $211.6 \pm 1.4$ & $-14,3 \pm 0,9$ \\
$5 \%$ & $303.1 \pm 2.8$ & $-23,8 \pm 1,1$ \\
$10 \%$ & $375.0 \pm 3.8$ & $-27,2 \pm 0,8$ \\
$20 \%$ & $557.1 \pm 4.4$ & $-29,3 \pm 1,3$ \\
\hline
\end{tabular}

In Figure 3, the pretreatments of the PEI blocks with the $\mathrm{MG}$ were conducted at $C_{M G}=$ $4 \mathrm{mg} / \mathrm{mL}$ which we supposed was above the concentration necessary to reach saturation of the block surfaces. To confirm this hypothesis, we tested different pretreatments concentrations, for all the MG (MAA 5\%, 10\% and 20\%) ranging from $C_{M G}=0.008 \mathrm{mg} / \mathrm{mL}$ to $8 \mathrm{mg} / \mathrm{mL}$. In Figure 4A, the total aggregation \% (the total percentage of aggregated blocks independently of the aggregate size) is represented versus the MAA concentration in the microgel suspension during pretreatment $\left(C_{M A A}\right)$ after one aggregation experiment $(5$ separate repetitions). We observed that aggregation of the hydrogel blocks did not occur below a critical concentration, $C_{M A A}^{*}$, which increased with the MAA content in the microgels, from $2.9 \mu \mathrm{g} / \mathrm{mL}$ for MAA $5 \%$ to $11.7 \mu \mathrm{g} / \mathrm{mL}$ for MAA $20 \%$. Interestingly, these values of $C_{M A A}^{*}$ corresponded to a similar microgel concentration, $C_{M G}^{*}=0.04 \mathrm{mg} / \mathrm{mL}$ for all the $\mathrm{MG}$. 

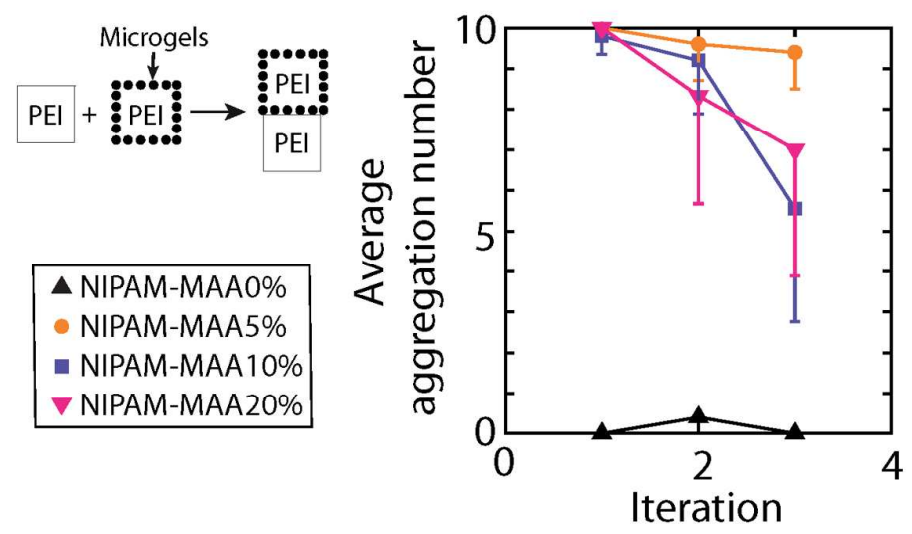

Figure 3: Effect of MAA content in microgels on the directed assembly of PEI-containing hydrogel blocks. In contrast to the data shown in Figure 2, the average aggregation number of this system only slightly decreases with the number of assembly/disassembly iteration. Error bars represent the standard deviation of 5 separate experiments. Curves are guides for the eye.

One possible explanation of such behavior is that the microgel size increases significantly with the MAA content (Table 1) without any significant changes in zeta potential. Therefore, most of the MAA is expected to be located inside the microgel particle and not at its surface.

242 Consequently, the charge surface density is expected to decrease with the MAA\% in the microgel which could explain why $C_{\mathrm{MAA}}{ }^{*}$ was found to increase with MAA\%. In Figure 4B-D, we show the evolution of the average aggregation number as a function of the assembly/disassembly iteration number for each microgel concentration used. In these panels, the indicated concentration of microgels corresponds to the microgel concentration in the pretreatment suspension. Results show a quasi-constant (or slightly decreasing) average aggregation number for $C_{\mathrm{MG}}>0,08 \mathrm{mg} / \mathrm{mL}$ and complete loss of blocks aggregation when $C_{\mathrm{MG}}<0.08 \mathrm{mg} / \mathrm{mL}$. Results were identical for MAA5\%, 10\% and 20\% microgel pretreatments. We also noticed that pretreatment with the MAA $20 \%$ microgels at $0.08 \mathrm{mg} / \mathrm{mL}$ systematically lead to significantly smaller aggregates compared to the other MG at the

252 same $C_{M G}$. Observation of a similar aggregate distribution and average aggregation number at $253 C_{M G}$ superior to $0.08 \mathrm{mg} / \mathrm{ml}$, independently of the MAA content in the microgels, tends to 
255 concentrations. In Supplementary information 3, the evolution of the cumulative percentage 256 of aggregated cubes as a function of the aggregation number confirmed that the size 257 distribution of the aggregates was not affected at $C_{\mathrm{MG}}>0.08 \mathrm{mg} / \mathrm{mL}$.
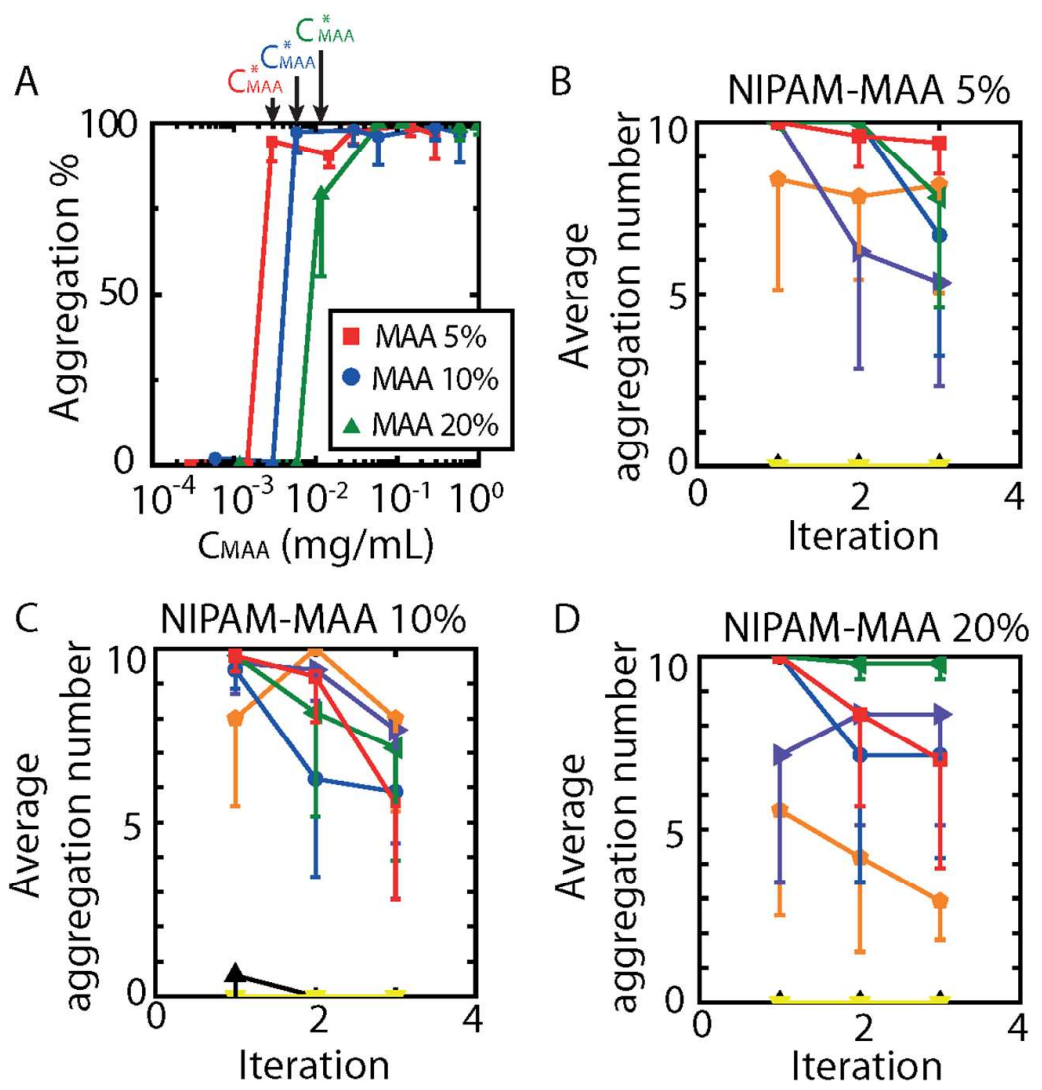

$$
\begin{array}{|c|c|}
\hline C_{M G}= & 0,4 \mathrm{mg} / \mathrm{mL} \\
\Delta 0,008 \mathrm{mg} / \mathrm{mL} & 0,8 \mathrm{mg} / \mathrm{mL} \\
0,04 \mathrm{mg} / \mathrm{mL} & 4 \mathrm{mg} / \mathrm{mL} \\
0,08 \mathrm{mg} / \mathrm{mL} & =8 \mathrm{mg} / \mathrm{mL} \\
\hline
\end{array}
$$

Figure 4: Effect of microgel concentration on the directed assembly of PEI blocks. A) Blocks aggregation $\%$ as a function of the MAA content in the microgels during the pretreatment. BD) Average blocks aggregation number as a function of the experiment iteration. Error bars represent the standard deviation of 5 separate experiments. Lines are guides for the eye.

259

260 To understand the role played by the electrostatic forces in the assembly of the hydrogel

261 blocks, we performed a series of aggregation tests at increasing salt concentrations $\left(C_{\text {Salt }}=0\right.$ -

$262150 \mathrm{mM} \mathrm{NaCl}$, see Figure 5A). Above a critical salt concentration, $C_{\text {Salt }}^{*}$, the aggregation of the 
263 hydrogel blocks was strongly hampered, independently of the system. PEI/HA aggregates

264 were found to resist significantly more to the increase in salinity $\left(C_{\text {Salt }}^{*}=80 \mathrm{mM}\right.$ ), even after 265 prolonged incubation in saline solution (24h) compared to PEI/MG systems. The value 266 of $C_{\text {Salt }}^{*}$ was found to depend on the MG composition, and increased with the MAA content, 267 from $C_{\text {Salt }}^{*}=5 \mathrm{mM}$ for MAA $5 \%$ to $C_{\text {Salt }}^{*}=20 \mathrm{mM}$ for MAA $20 \%$. The value of $C_{\text {Salt }}^{*}$ was 268 also found to depend on the $C_{M G}$ (See Figure 5B). While the pretreatments with $C_{M G}=8$ and $2694 \mathrm{mg} / \mathrm{mL}$ presented similar behavior $\left(C_{\text {Salt }}^{*}=20 \mathrm{mM}\right)$, a decrease in $C_{\text {Salt }}^{*}$ was observed at $C_{M G}=$ $2700.4 \mathrm{mg} / \mathrm{mL}\left(C_{\text {Salt }}^{*}=10 \mathrm{mM}\right)$ until almost complete loss of directed assembly was observed at $271 C_{M G}=0.08 \mathrm{mg} / \mathrm{mL}\left(C_{\text {Salt }}^{*}=2.5 \mathrm{mM}\right)$.
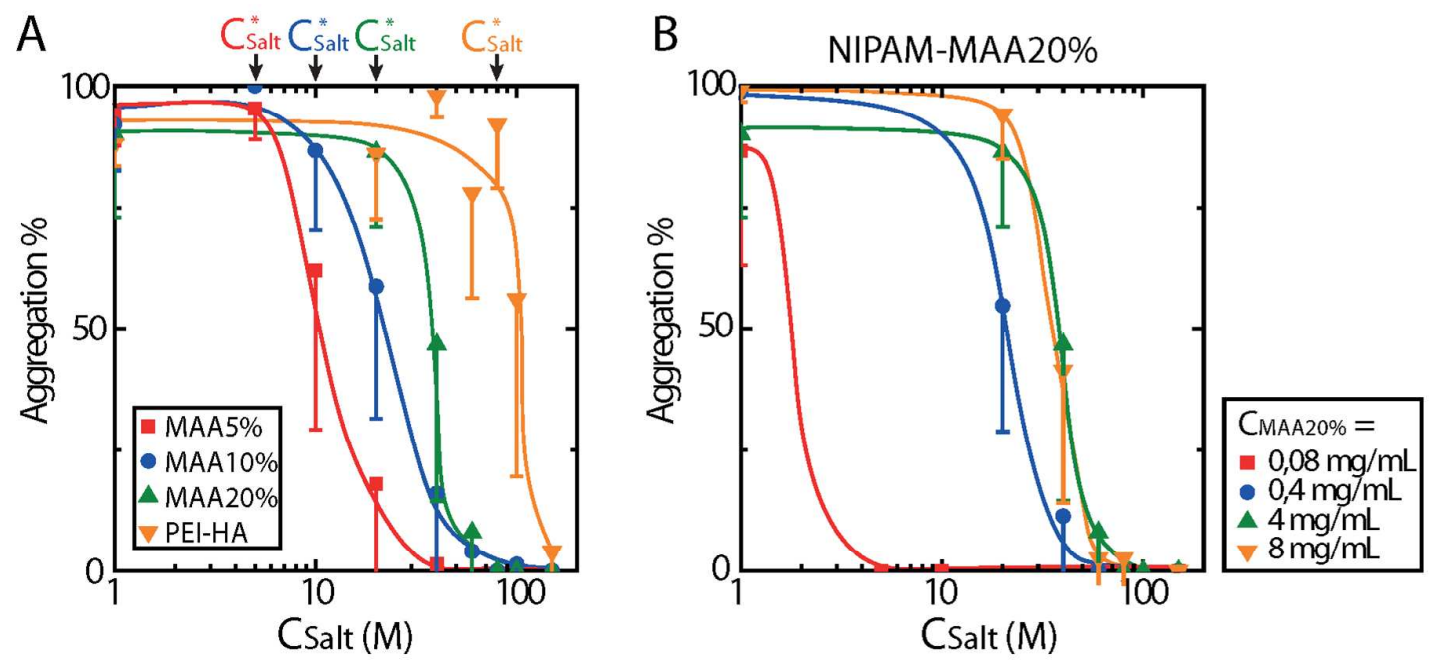

Figure 5: Effect of salt concentration on the directed assembly of A) PEI/HA and PEI/MG systems B) PEI/MG system in presence of microgels NIPAM-MAA20\% at different concentrations. Lines are guides for the eye.

272

273 Since the two systems under study are composed of $\mathrm{pH}$-sensitive materials (PEI and HA), the

274 effect of $\mathrm{pH}$ on the directed assembly of the hydrogel blocks was also studied. We compared

275 our previous results obtained in pure water $(\mathrm{pH}=6)$ with tests performed in acidic $(\mathrm{pH}=3)$

276 and basic $(\mathrm{pH}=10.5)$ conditions (see Figure 6). Results show a complete loss of assembly at

277 a $\mathrm{pH}$ above or below $\mathrm{pH}=6$ after $24 \mathrm{~h}$ of equilibrium. 


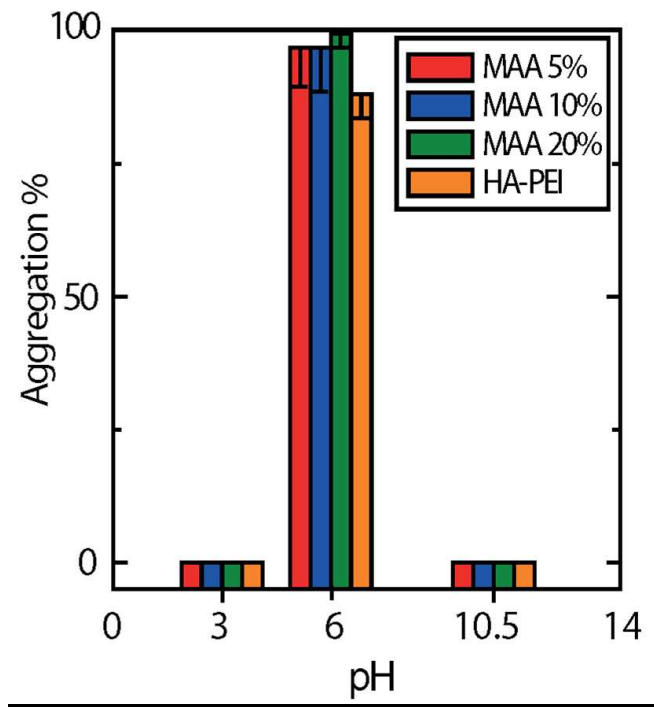

Figure 6: Effect of $\mathrm{pH}$ on the directed assembly (aggregation) of PEI/HA and PEI/MG systems

\section{Discussion}

Macroscopic observations of the blocks aggregates pointed out differences in interaction strength between PEI/HA and PEI/MG systems. Larger PEI/HA aggregates were indeed systematically observed compared to more compact PEI/MG aggregates. For the PEI/HA system, we observed that the blocks concentration (population size) played an important role and negative blocks did not necessarily lead to an adhesive contact. Nevertheless, only adhesive contacts between positive and negative blocks were observed, demonstrating that directed assembly, in opposition to self-assembly, was effectively happening. The total loss of assembly capacity of the PEI/HA blocks after a few iterations strongly suggest that the hydrogel blocks surfaces are very sensitive to mechanical manipulation and therefore prone to damage. Surface damage can occur in the form of surface roughening or material transfer between surfaces (which leads to surface charge compensation), both causes leading to adhesion loss and consequently to a decrease of the aggregation number. Those initials observations suggested that in the case of the PEI/HA system, adhesive contacts are mostly 
294 promoted by steric entanglements and electrostatic interactions between polyelectrolytes 295 chains present at the hydrogel blocks surfaces (See Figure 7A).

296 We observed that the effect of $C_{\text {Salt }}$ on the blocks aggregation is not gradual, meaning that the 297 aggregates size did not continuously decrease with salt concentration. Instead, an abrupt 298 transition from an aggregated to a disaggregated state was observed around $C_{\text {Salt }}^{*}$. 299 Surprisingly, $C_{\text {Salt }}^{*}$ was found to be much higher for the HA/PEI system compared to the $300 \mathrm{PEI} / \mathrm{MG}$ system suggesting that other than purely electrostatic forces might be at work in this 301 system. In fact, the large variability in the assembly process of HA-PEI blocks and the high $302 C_{\text {Salt }}^{*}$ value indicate that electrostatic and macromolecular entanglements are involved in the 303 adhesion mechanism.

304 Since HA and PEI are polyelectrolytes, their ionization degree is directly determined by the $305 \mathrm{pH}$ of the medium. HA possess carboxylic acid groups with a $\mathrm{pKa} \approx 3-4^{36}$. While HA chains 306 are only partially negatively charged at $\mathrm{pH}=3(24 \%$ ionization, $\mathrm{pH} \approx \mathrm{pKa})$, at $\mathrm{pH}=6$ and 10 307 HA is fully neutralized $(\mathrm{pH}>\mathrm{pKa})$. On the other hand, branched PEI possess primary, 308 secondary and tertiary amines and therefore three respective $\mathrm{pKa}\left(4.5,6.7\right.$ and $\left.11.6^{37}\right)$. Using 309 the structure of the branched PEI used in this study (primary:secondary:tertiary amines ratio 310 of $4: 3: 4$ ), the total amount of ionized amine groups (in form of $\mathrm{NH}^{+}, \mathrm{NH}_{2}^{+}$and $\mathrm{NH}_{3}^{+}$) 311 available on the PEI chains at a given $\mathrm{pH}$ can be estimated. At $\mathrm{pH}=3,98.9 \%$ of the amine 312 groups are positively charged, $60.2 \%$ at $\mathrm{pH}=6$ (secondary and tertiary amines) and only $31333.7 \%$ at $\mathrm{pH}=10.5$ (tertiary amines only). To insure rapid adhesive electrostatic interactions 314 between block surfaces, negative and positive surfaces must be significantly ionized. This 315 explained why assembly was only observed at $\mathrm{pH}=6$. At this $\mathrm{pH}$, blocks exposed significant 316 amount of charged groups $(99.7 \%$ for $\mathrm{HA}$ and $60.2 \%$ for $\mathrm{PEI})$, which was not the case at $\mathrm{pH}=$ 3173 (24\% HA ionization) and 10.5 (33.7\% PEI ionization). Moreover, polyelectrolyte charge 318 has an effect on chains conformation. At high ionization degree, polymer chains from one 
block's surface are expected to expand which favors overlapping and entanglement upon contact with another surface and therefore adhesion. Our study shows that assembly of

321 hydrogel blocks can occur at partial ionization of the polyelectrolytes ( $60 \%$ for PEI) but can

322 be inhibited if ionization is too small (the minimum being located between 30 and 60\%).

As for the PEI/MG systems, MGs were found to act as efficient adhesion promoters between positively charged blocks. By electrostatically interacting with the PEI chains and potentially the network of HEMA-PEGDMA forming the blocks, MGs can create a negatively charged 7B). Since the MGs are significantly more crosslinked than the hydrogel blocks, interpenetration between polyelectrolytes chains and MGs is expected to be disfavored. Therefore, microgel adsorption and blocks adhesion are both driven by MAA groups at the surface of the microgel. This explanation is also confirmed by the fact that no directed assembly with MGs of pure NIPAM (which were found to be slightly charged) was observed.

One major difference between the PEI/MG and the PEI/HA systems is the surprisingly good assembly reproducibility after several iterations of the PEI/MG system (See Figure 3). The most straightforward explanation of this observation is the absence of damage under mechanical manipulation of the blocks, and a perfectly reversible interactions between PEI blocks and MGs. This would suggest that MGs adsorbed at the blocks surfaces are compliant and can easily move on the gel interface to adapt their conformation and avoid surface damage.

The effect of the ionic strength seems to be modulated by the composition of the microgels. The critical concentration at which assembly was inhibited, $C_{\text {Salt }}^{*}$, was found to increase strongly with the MAA\% in the microgels from $5 \mathrm{mM}$ for MAA $5 \%$ to $20 \mathrm{mM}$ for MAA $20 \%$. This observation confirms the crucial role of MAA moieties on the interactions between block surfaces. The disrupting effect of $\mathrm{NaCl}$ is explained by the hindering of the interactions 
344 between MAA at microgel surfaces and PEI chains (See Figure 5A). Higher MAA content in

345 the MGs explain the increased $C_{\text {Salt }}^{*}$ as more chloride anions are needed to completely screen

346 PEI-microgels interactions. Stability tests also confirmed that microgels were stable at very

347 high $C_{\text {Salt }}$ (data not shown). The critical coagulation concentrations of the microgels are

348 indeed significantly higher than the $C_{\text {Salt }}$ used in our tests meaning that the microgels

349 remained as a stable colloidal suspension and, at least in part, electrostatically charged and

350 thus prone to interactions with PEI chains. Loss of directed assembly could also be due to PEI

351 polyelectrolytes chains reorganization and folding, decreasing possible interactions with the

352 MGs.

353 The influence of $\mathrm{pH}$ on the directed assembly of PEI/MG is quite similar to PEI/HA system.

354 Linear MAA chains with a degree of polymerization superior to 20, present a pKa of $6.5^{39}$.

355 Considering this information, microgels should exhibit no ionization of the MAA at $\mathrm{pH}=3$ and

356 complete ionization at $\mathrm{pH}=10.5(>99.9 \%)$. Therefore, in acidic or basic conditions, MAA

357 and PEI are not ionized enough to favor electrostatic interactions. At $\mathrm{pH}=6$, MAA presents

$35824.0 \%$ of ionization which seems sufficient to promote interactions with the charged amines

359 of the PEI. However, the fate of the microgels after PEI/MG block equilibrations at $\mathrm{pH}=3$

360 and 10.5 solutions remains unknown. It is indeed unclear if microgels remained adsorbed or

361 entrapped in the HEMA-PEDGMA and PEI networks or if they were released upon loss of

362 ionization.

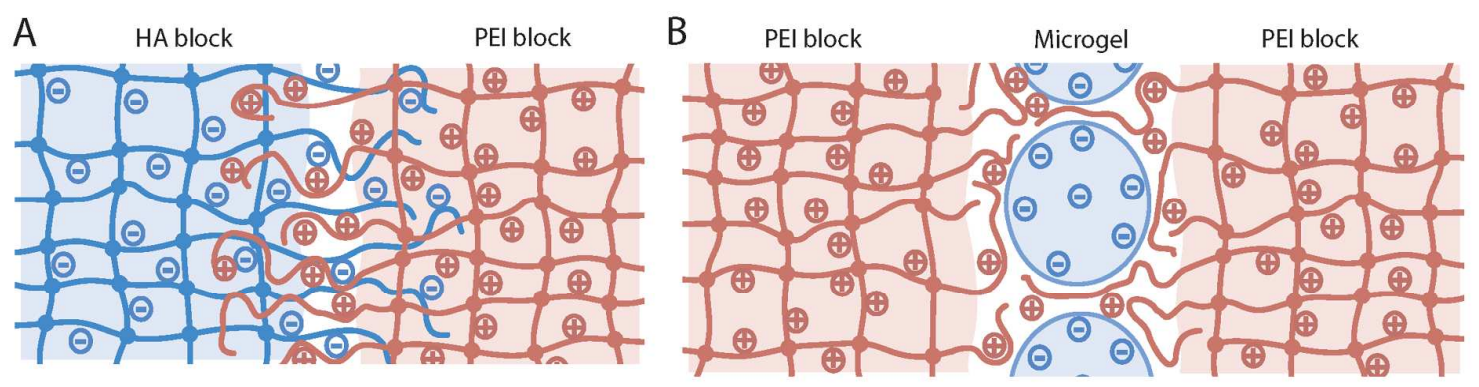

Figure 7: Models of supposed interactions at hydrogel blocks surfaces during adhesive contacts, A: steric entanglement guided by electrostatic cues between HA and PEI 
polyelectrolytes chains, B: bridging between PEI chains and NIPAM-MAA microgels without any entanglements involved.

363

364

In summary, the assembly of PEI/HA blocks were found to be driven by electrostatic interactions and steric entanglements. As a consequence, this system was prone to surface damage upon repeated forced desaggregation. The PEI/MG system is based on the reversible electrostatic interactions between ionized MAA groups in the MGs and PEI polyelectrolytes chains. This systems was not damaged under mechanical manipulation but was highly sensitive to ionic strength. These observations highlight the important, and overlooked role of the interface microstructure in the adhesion mechanism (see Figure 7). Hydrogel-hydrogel interfaces in presence of microgels are expected to be rougher compared to direct hydrogelhydrogel contacts allowing for ions to quickly penetrate the interface and to destabilize it even if the adhesive strength between blocks is stronger.

\section{Conclusions}

This study presents the directed assembly of charged hydrogel blocks mediated by microgel particles or by direct contact. In both systems studied, random contacts between blocks resulted in the formation of aggregates. PEI/HA directed assembly in water resulted in large, flexible aggregates vulnerable to mechanical manipulation, while PEI/MG aggregates were more compact and resistant. Such difference was attributed to a difference in adhesion strength between blocks. The PEI/MG system presented the highest sensitivity to ionic strength, highlighting the role of the interface microstructure and porosity in the adhesion phenomena.

These results provide new insights into the adhesion mechanism between soft materials in presence of a third body such as microgels, proteins or solid nanoparticles and should guide the development of future materials with controlled tunable properties. 
387

388

389

390

391

392

393

394

395

396

397

398

399

400

401

402

403

404

405

406

407

408

409

410

411

412

413

414

415

416

417

418

419

420

421

422

423

424

425

426

427

428

429

\section{Acknowledgements}

$\mathrm{XB}$ is grateful for the financial support of FRQ-NT (new researcher program) and CRC. NH

acknowledges the financial support of the Faculty of Pharmacy (recruitment scholarship).

PLL is grateful for financial support of GRUM, Faculty of Pharmacy, FRQ-NT and NSERC.

\section{References}

1. Hoffman, A. S. Hydrogels for biomedical applications. Adv. Drug Del. Rev. 2012,64, Supplement, 18-23.

2. King, W. J.; Krebsbach, P. H. Growth factor delivery: How surface interactions modulate release in vitro and in vivo. Adv. Drug Del. Rev. 2012,64 (12), 1239-1256.

3. Hanauer, N.; Latreille, P.; Alsharif, S.; Banquy, X. 2D, 3D and 4D Active Compound Delivery in Tissue Engineering and Regenerative Medicine. Curr. Pharm. Des. 2015.

4. Hoare, T. R.; Kohane, D. S. Hydrogels in drug delivery: progress and challenges. Polymer 2008,49 (8), 1993-2007.

5. Priya, S. G.; Jungvid, H.; Kumar, A. Skin tissue engineering for tissue repair and regeneration. Tissue Eng. Pt B-Rev 2008,14 (1), 105-118.

6. Metcalfe, A. D.; Ferguson, M. W. J. Bioengineering skin using mechanisms of regeneration and repair. Biomaterials 2007,28 (34), 5100-5113.

7. Lam, J.; Clark, E. C.; Fong, E. L. S.; Lee, E. J.; Lu, S.; Tabata, Y.; Mikos, A. G. Evaluation of cellladen polyelectrolyte hydrogels incorporating poly(I-Lysine) for applications in cartilage tissue engineering. Biomaterials 2016,83, 332-346.

8. Muzzarelli, R. A. A.; Greco, F.; Busilacchi, A.; Sollazzo, V.; Gigante, A. Chitosan, hyaluronan and chondroitin sulfate in tissue engineering for cartilage regeneration: A review. Carbohydrate Polymers 2012,89 (3), 723-739.

9. Griffin, K. S.; Davis, K. M.; McKinley, T. O.; Anglen, J. O.; Chu, T.-M. G.; Boerckel, J. D.; Kacena, M. A. Evolution of Bone Grafting: Bone Grafts and Tissue Engineering Strategies for Vascularized Bone Regeneration. Clin. Rev. Bone. Miner. Metab. 2015,13 (4), 232-244.

10. Yang, J.-A.; Yeom, J.; Hwang, B. W.; Hoffman, A. S.; Hahn, S. K. In situ-forming injectable hydrogels for regenerative medicine. Prog. Polym. Sci. 2014,39 (12), 1973-1986.

11. Shu, X. Z.; Liu, Y.; Palumbo, F. S.; Luo, Y.; Prestwich, G. D. In situ crosslinkable hyaluronan hydrogels for tissue engineering. Biomaterials 2004,25 (7), 1339-1348.

12. Cai, S.; Liu, Y.; Shu, X. Z.; Prestwich, G. D. Injectable glycosaminoglycan hydrogels for controlled release of human basic fibroblast growth factor. Biomaterials 2005,26 (30), 6054-6067.

13. Tsukuda, Y.; Onodera, T.; Ito, M.; Izumisawa, Y.; Kasahara, Y.; Igarashi, T.; Ohzawa, N.; Todoh, M.; Tadano, S.; Iwasaki, N. Therapeutic effects of intra-articular ultra-purified low endotoxin alginate administration on an experimental canine osteoarthritis model. Journal of Biomedical Materials Research Part A 2015,103 (11), 3441-3448.

14. Kim, G. O.; Kim, N.; Kim, D. Y.; Kwon, J. S.; Min, B.-H. An electrostatically crosslinked chitosan hydrogel as a drug carrier. Molecules 2012,17 (12), 13704-13711.

15. Salem, A. K.; Rose, F. R.; Oreffo, R. O.; Yang, X.; Davies, M. C.; Mitchell, J. R.; Roberts, C. J.; Stolnik-Trenkic, S.; Tendler, S. J.; Williams, P. M. Porous polymer and cell composites that self-assemble in situ. Adv. Mater. 2003,15 (3), 210-213.

16. Miyata, T.; Asami, N.; Uragami, T. Preparation of an antigen-sensitive hydrogel using antigenantibody bindings. Macromolecules 1999,32 (6), 2082-2084. 
17. Sant, S.; Coutinho, D. F.; Sadr, N.; Reis, R. L.; Khademhosseini, A. Tissue Analogs by the Assembly of Engineered Hydrogel Blocks. Biomimetic Approaches for Biomaterials Development 2012, 471-493.

18. Whitesides, G. M.; Boncheva, M. Beyond molecules: Self-assembly of mesoscopic and macroscopic components. Proceedings of the National Academy of Sciences 2002,99 (8), 4769-4774.

19. Cheng, H.-w.; Luk, K. D. K.; Cheung, K. M. C.; Chan, B. P. In vitro generation of an osteochondral interface from mesenchymal stem cell-collagen microspheres. Biomaterials 2011,32 (6), 1526-1535.

20. Tan, W.; Desai, T. A. Layer-by-layer microfluidics for biomimetic three-dimensional structures. Biomaterials 2004,25 (7-8), 1355-1364.

21. McGuigan, A. P.; Sefton, M. V. Vascularized organoid engineered by modular assembly enables blood perfusion. Proceedings of the National Academy of Sciences 2006,103 (31), 1146111466.

22. Chung, S. E.; Park, W.; Shin, S.; Lee, S. A.; Kwon, S. Guided and fluidic self-assembly of microstructures using railed microfluidic channels. Nature materials 2008,7 (7), 581-587.

23. Du, Y.; Lo, E.; Ali, S.; Khademhosseini, A. Directed assembly of cell-laden microgels for fabrication of 3D tissue constructs. Proceedings of the National Academy of Sciences of the United States of America 2008,105 (28), 9522-9527.

24. Fernandez, J. G.; Khademhosseini, A. Micro-masonry: construction of 3D structures by microscale self-assembly. Adv. Mater. 2010,22 (23), 2538-2541.

25. Xu, M.; Wang, X.; Yan, Y.; Yao, R.; Ge, Y. An cell-assembly derived physiological 3D model of the metabolic syndrome, based on adipose-derived stromal cells and a gelatin/alginate/fibrinogen matrix. Biomaterials 2010,31 (14), 3868-3877.

26. Ekici, S.; Ilgin, P.; Yilmaz, S.; Aktas, N.; Sahiner, N. Temperature and magnetic field responsive hyaluronic acid particles with tunable physical and chemical properties. Appl. Surf. Sci. 2011,257 (7), 2669-2676.

27. Liu, B.; Liu, Y.; Lewis, A. K.; Shen, W. Modularly assembled porous cell-laden hydrogels. Biomaterials 2010,31 (18), 4918-4925.

28. Harada, A.; Kobayashi, R.; Takashima, Y.; Hashidzume, A.; Yamaguchi, H. Macroscopic selfassembly through molecular recognition. Nature chemistry 2011,3 (1), 34-37.

29. Yamaguchi, H.; Kobayashi, Y.; Kobayashi, R.; Takashima, Y.; Hashidzume, A.; Harada, A. Photoswitchable gel assembly based on molecular recognition. Nature communications 2012,3, 603.

30. Qi, H.; Ghodousi, M.; Du, Y.; Grun, C.; Bae, H.; Yin, P.; Khademhosseini, A. DNA-directed selfassembly of shape-controlled hydrogels. Nature communications 2013,4.

31. Gillette, B. M.; Jensen, J. A.; Tang, B.; Yang, G. J.; Bazargan-Lari, A.; Zhong, M.; Sia, S. K. In situ collagen assembly for integrating microfabricated three-dimensional cell-seeded matrices. Nature materials 2008,7 (8), 636-640.

32. Yu, Y.; Kieviet, B. D.; Kutnyanszky, E.; Vancso, G. J.; de Beer, S. Cosolvency-induced switching of the adhesion between poly (methyl methacrylate) brushes. ACS macro letters 2014,4 (1), 75-79.

33. Cao, Z.; Dobrynin, A. V. Nanoparticles as Adhesives for Soft Polymeric Materials. Macromolecules 2016,49 (9), 3586-3592.

34. Rose, S.; Prevoteau, A.; Elzière, P.; Hourdet, D.; Marcellan, A.; Leibler, L. Nanoparticle solutions as adhesives for gels and biological tissues. Nature 2014,505 (7483), 382-385.

35. Brunel, B.; Beaune, G.; Nagarajan, U.; Dufour, S.; Brochard-Wyart, F.; Winnik, F. M. Nanostickers for cells: a model study using cell-nanoparticle hybrid aggregates. Soft Matter 2016,12 (38), 7902-7907.

36. Mero, A.; Campisi, M. Hyaluronic acid bioconjugates for the delivery of bioactive molecules. Polymers 2014,6 (2), 346-369.

37. Demadis, K. D.; Paspalaki, M.; Theodorou, J. Controlled release of bis (phosphonate) pharmaceuticals from cationic biodegradable polymeric matrices. Industrial \& Engineering Chemistry Research 2011,50 (9), 5873-5876. 
481 38. Fuller, K.; Tabor, D. In The effect of surface roughness on the adhesion of elastic solids, 482 Proceedings of the Royal Society of London A: Mathematical, Physical and Engineering Sciences, 483 1975; The Royal Society, pp 327-342.

484 39. Izumrudov, V. A.; Kharlampieva, E.; Sukhishvili, S. A. Multilayers of a globular protein and a 485 weak polyacid: role of polyacid ionization in growth and decomposition in salt solutions. 486 Biomacromolecules 2005,6 (3), 1782-1788.

487

488 
489 TOC Graphic

490
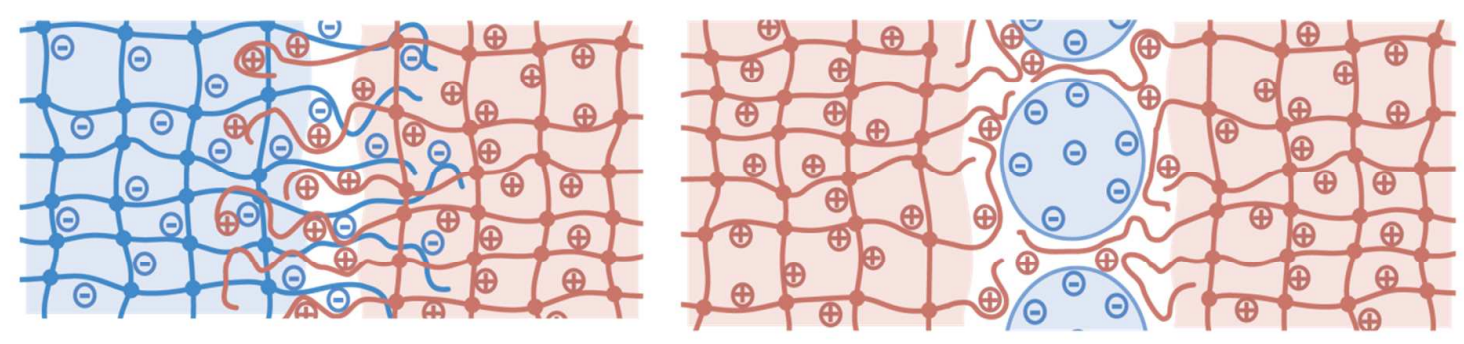

14

14
15
16

16

17

18

19

20

21

22

23

24

25

26

27

28

29

30

31

32

33

34

35

36

37

38

39

40

41

42

43

44

45

46

47

48

49

50

51

52

53

54

55

56

57

58

59

60 